\title{
Анализ механизмов уплотнения термоэлектрических порошков скуттерудитов и сплавов Гейслера в процессе активированного полем спекания
}

\author{
(C) А.С. Тукмакова ${ }^{1}$, Н.И. Хахилев ${ }^{1}$, Д.Б. Щеглова ${ }^{1}$, В.Д. Насонов ${ }^{1}$, А.П. Новицкий ${ }^{2}$, \\ И.А. Сергиенко ${ }^{2}$ А.В. Новотельнова ${ }^{1}$ \\ ${ }^{1}$ Национальный исследовательский университет ИТМО, \\ 197101 Санкт-Петербург, Россия \\ ${ }^{2}$ Национальный исследовательский технологический университет „МИСиС“, \\ 119049 Москва, Россия \\ E-mail: astukmakova@itmo.ru, daria.shcheglova1998@gmail.com
}

Поступила в Редакцию 12 августа 2021 г.

В окончательной редакции 28 августа 2021 г.

Принята к публикации 28 августа 2021 г.

Для расчета параметров уплотнения термоэлектрических порошков скуттерудитов на основе $\mathrm{CoSb}_{3}$ и сплавов Гейслера на основе $\mathrm{Fe}_{2} \mathrm{VAl}$ был проведен анализ скорости усадки порошков, основанный на модели вязкотекучей деформации пористого тела. Показано, что этот подход, ранее использовавшийся для порошков металлов и керамик, также применим для термоэлектрических порошков. Получены значения чувствительности материала к скорости деформации, на основе которых определены соответствующие механизмы уплотнения порошков. Для скуттерудитов было выявлено преобладание механизма ползучести, контролируемого скольжением дислокаций, с дальнейшим переходом к механизмам проскальзывания зерен и диффузионной ползучести. Для образцов сплавов Гейслера основным механизмом являлась ползучесть за счет проскальзывания зерен, переходящая к диффузионной ползучести.

Ключевые слова: активированное полем спекание, численное моделирование, усадка порошка, скуттерудиты, сплавы Гейслера.

DOI: $10.21883 /$ FTP.2021.12.51695.10

\section{1. Введение}

Термоэлектрическая добротность материала в значительной степени зависит от величины теплопроводности, которую можно снизить при помощи создания объемных наноструктурированных материалов. Наиболее эффективным способом для достижения такой структуры материала является активированное полем спекание (АПС, Field-Assisted Sintering Technique/FAST), при котором к нанопорошку прикладывается внешнее одноосное давление и пропускается импульсный переменный или постоянный ток [1]. Это обеспечивает образование теплоты Джоуля, нагрев и компактирование порошка. Конечная структура компактированного материала определяет будущие термоэлектрические свойства.

Для анализа процесса АПС необходимо учитывать две группы данных: 1) технологические параметры процесса и 2) данные о микроструктуре образца (например, гранулометрический состав до процесса спекания или размер зерна после). Технологические параметры отслеживаются на протяжении всего процесса, к ним относятся: температура, скорость нагрева, давление, сила электрического тока и изменение высоты образца. В то время как данные о структуре образца могут быть отслежены только до процесса и после него. К характеристикам порошка до процесса спекания относятся: гранулометрический состав, насыпная плотность, результаты рентгеноструктурного анализа. Данные о структуре образца после спекания включают: пористость (или относительную плотность), размер зерна, изменение фазового состава, наличие областей вторичной рекристаллизации, характеристики межзеренных границ, дислокаций и т.д.

Данные, которые затруднительно получить экспериментально и которых не хватает для целостного понимания картины АПС, - это изменение микроструктуры материала на протяжении уплотнения порошка. Восстановление картины кинетики спекания возможно на основе анализа скорости усадки образца (производной пористости по времени) [2] с применением модели вязкотекучей деформации пористого тела [3]. Основная идея данного подхода заключается в том, что численная оценка скорости усадки порошка позволяет определить механизмы компактирования: различные типы движения дислокаций, зерен, либо диффузионные процессы.

Существует ряд работ, посвященных исследованию уплотнения керамических нанопорошков. Например, в работе Вэя и др. [2] был исследован процесс усадки порошка $\mathrm{ZrC}$ при АПС при температурах $1650-2100^{\circ} \mathrm{C}$. Было выяснено, что основными механизмами уплотнения являлись проскальзывание границ зерен и ползучесть при скольжении дислокаций. В работе Ли и др. было проведено исследование механизмов уплотнения $\mathrm{ZrN}$ при АПС и высоковольтной электроимпульсной 
консолидации [4]. Было выявлено, что при АПС доминирующим механизмом уплотнения является ползучесть, обусловленная переползанием дислокаций (dislocation climb). Однако при приложении высокого электрического напряжения механизм менялся на диффузионную ползучесть. В [5] в качестве основного механизма уплотнения $\mathrm{ZrN}$ при АПС была выделена ползучесть за счет скольжения дислокаций (dislocation glide), в то время как горячее прессование при таких же температуре и давлении характеризовалось преобладанием ползучести, обусловленной переползанием дислокаций. Основной механизм уплотнения зависит от значения температуры спекания и прикладываемого давления. Например, для порошка $\mathrm{B}_{4} \mathrm{C}$ [6] при температурах до $2000^{\circ} \mathrm{C}$ и давлении до 40 МПа преобладающим механизмом уплотнения было проскальзывание границ зерен, а при температурах выше $2000^{\circ} \mathrm{C}$ и давлении выше 40 МПа основным механизмом являлась ползучесть, контролируемая переползанием дислокаций.

Также достаточно хорошо изучены механизмы уплотнения нанопорошков металлов при АПС. Для Та была выявлена аналогичная $\mathrm{B}_{4} \mathrm{C}$ зависимость основного механизма уплотнения от температуры и давления [7]. Было показано, что увеличение температуры приводит к смене основного механизма деформации с зернограничной диффузии на ползучесть, контролируемую переползанием дислокаций. Для TiAl [8] основным механизмом уплотнения является ползучесть при переползании дислокаций, сопровождаемая диффузией $\mathrm{Al}$, а ползучесть при скольжении дислокаций характерна только для начала процесса усадки.

Дополнение уравнения вязкотекучей деформации с учетом влияния электромиграции при спекании металлического нанопорошка вольфрама было использовано в работе Денга и др. [9]. В исследовании было показано, что увеличение плотности тока способствует уменьшению энергии активации вязкотекучей деформации. Также процесс уплотнения был условно разделен на две стадии: при более низком давлении основным механизмом уплотнения является зернограничная диффузия, при более высоком - ползучесть при переползании дислокаций совместно с зернограничной диффузией. Немного другой подход был использован в исследовании Янга и др. [10], где усадка порошка описывалась через поверхностную энергию, средний размер частиц и вязкость порошка. Основываясь на значениях данных величин, авторы выделили 3 этапа уплотнения: усадка, контролируемая поверхностной энергией, дефектами кристаллической решетки и высокотемпературной ползучестью.

Так как все опубликованные работы написаны для порошков металлических и керамических материалов, встает вопрос о возможности применения данного подхода к анализу спекания полупроводниковых термоэлектрических порошков, обладающих отличающимися свойствами. В данной работе выбранная методика применяется к двум группам среднетемпературных термоэлектриков: сплавам Гейслера на основе $\mathrm{Fe}_{2} \mathrm{VAl}$ и скуттерудитам на основе $\mathrm{CoSb}_{3}$. Цель данной работы - подтверждение целесообразности применения используемого подхода для анализа кинетики спекания термоэлектрических порошков.

\section{2. Анализ скорости усадки порошков с применением модели вязкотекучей деформации пористого тела}

Основываясь на уравнении вязкотекучей деформации [11], рассматривая пористое тело, подверженное приложенному извне давлению, Олевский вывел определяющее уравнение для явного описания напряженнодеформированного состояния во время спекания пористых материалов в следующем виде [12]:

$$
\sigma_{i j}=A_{c r} W^{m-1}\left[\varphi \dot{\varepsilon}_{i j}+\left(\psi-\frac{1}{3} \varphi\right) \dot{e} \delta_{i j}\right]+P_{L} \delta_{i j},
$$

где $\sigma_{i j}-$ внешнее давление; $W-$ эквивалентная эффективная скорость деформации, зависящая от пористости $(\theta)$, скоростей изменения формы и объема $(\dot{e})$, модулей сдвига $(\varphi)$ и упругости $(\psi) ; P_{L}-$ эффективное давление спекания, $\delta_{i j}$ - дельта Кронекера $\left(\delta_{i j}=1\right.$, если $i=j, \delta_{i j}=0$, если $\left.i \neq j\right)$; последним слагаемым в уравнении (1) в случае активированного полем спекания можно пренебречь; $\dot{\varepsilon}_{i j}-$ скорость деформации; $A_{c r}$ - коэффициент ползучести, зависящий от коэффициента $A_{m}$, являющегося комплексным параметром материала:

$$
A_{c r}=A_{m} T^{m} \exp \left(\frac{m Q}{R T}\right),
$$

где $T$ - температура, $m$ - чувствительность к скорости деформации, $Q-$ энергия активации вязкотекучей деформации, $R$ - газовая постоянная.

С использованием уравнения (1) было получено следующее уравнение [2]:

$$
\dot{\theta}=\frac{d \theta}{d t}=-\left(\frac{\sigma_{z}}{A_{m} T^{m} \exp \left(\frac{m Q}{R T}\right)}\right)^{\frac{1}{m}}\left(\frac{3 \theta}{2}\right)^{\frac{m+1}{2 m}}(1-\theta)^{\frac{m-3}{2 m}},
$$

где $\sigma_{z}$ - приложенное осевое напряжение, $\dot{\theta}-$ скорость изменения пористости.

В зависимости от температуры и прикладываемого давления выделяют следующие механизмы ползучести [13]:

- ползучесть дислокаций, подразделяемая на скольжение дислокаций (dislocation glide, движение дислокаций вдоль определенного кристаллографического направления) и переползание дислокаций (dislocation climb, преодоление дислокациями препятствий или вакансий); 
- диффузионная ползучесть (diffusional creep) - деформация кристаллических тел, вызванная диффузией вакансий в кристаллической решетке, приводящая к пластической деформации в материале;

- проскальзывание границ зерен (зернограничная диффузия - grain boundary sliding/grain boundary diffusion), заключающееся в смещении зерен относительно друг друга при малой скорости деформации и высокой температуре.

Доминирующий механизм ползучести определяется значением коэффициента $n$ или обратного ему коэффициента чувствительности к скорости деформации $m[1]$. В таком случае, анализ кинетики спекания на основе уравнения (3) сводится к поиску значения коэффициента $m$. Различные механизмы ползучести характеризуются следующими значениями $m$ :

- при $m=1, n=1$ - диффузионная ползучесть, также называемая ползучестью Набарро-Герринга;

- при $m=0.5, n=2$ - ползучесть при проскальзывании границ зерен, предложенная Гифкинсом;

- при $m=0.33, n=3$ - ползучесть, контролируемая скольжением дислокаций, представленная Виртманом;

- при $m=0.22-0.33, n=3-5$ - ползучесть, контролируемая переползанием дислокаций, также описанная Виртманом.

Процесс уплотнения нанопорошка зависит не только от коэффициента $m$, но и от коэффициентов $A_{m}$ и $Q$, представленных в формуле (3). Теоретическая оценка или расчет этих коэффициентов является нетривиальной задачей; $Q$ в большинстве исследований находится графоаналитическим методом [6-8], а $A_{m}$, характеризующий микроструктуру материала, либо считается постоянным на протяжении всего процесса [4], либо подбирается [2] (различными методами подбора коэффициентов функции при ее известном значении), либо не рассчитывается вовсе. В данной работе при помощи метода обобщенного понижающего градиента происходит подбор всех трех коэффициентов, однако особое внимание уделяется именно параметру $m$, который в данном случае меняется на протяжении процесса спекания, что позволяет оценить постепенную смену основных механизмов уплотнения.

\section{3. Анализ скорости усадки порошков сплавов Гейслера на основе $\mathrm{Fe}_{2} \mathrm{VAl}$ и скуттерудитов на основе $\mathrm{CoSb}_{3}$}

Анализ скорости усадки порошка проводился на 6 образцах разных составов на основе сплавов Гейслера $\mathrm{Fe}_{2} \mathrm{~V}_{1-x} \mathrm{Nb}_{x} \mathrm{Al}_{1-y} \mathrm{Ga}_{y}$, где $x=y=0,0.1$ и 0.2 (табл. 1) и 7 образцах скуттерудитов номинального состава $\mathrm{Ga}_{x} \mathrm{In}_{y} \mathrm{Te}_{z} \mathrm{Co}_{4} \mathrm{Sb}_{12}$, где $x=y=0,1, \mathrm{a} z=0,0.5,1,2$, 3 (табл. 2).

Спекание образцов сплавов Гейслера и скуттерудитов производилось с использованием установки искрового плазменного спекания Dr. Sinter-1080 SPS (Fuji-SPS,
Таблица 1. Номинальный состав, масса до спекания $m_{\text {нач, }}$ диаметр пресс-формы $d_{\text {пф }}$, плотность после спекания $\rho_{\text {кон }}$, высота образцов до, $h_{0}$, и после, $h_{\text {кон }}$ спекания сплавов Гейслера $\mathrm{Fe}_{2} \mathrm{~V}_{1-x} \mathrm{Nb}_{x} \mathrm{Al}_{1-y} \mathrm{Ga}_{y}$ ( $x=y=0,0.1$ и 0.2 )

\begin{tabular}{l|c|c|c|c|c}
\hline Номинальный состав & $\begin{array}{c}m_{\text {нач},}, \\
\Gamma\end{array}$ & $\begin{array}{c}d_{\text {пф, }}, \\
\text { мм }\end{array}$ & $\begin{array}{c}\rho_{\text {кон }}, \\
\Gamma / \mathrm{cm}^{3}\end{array}$ & $\begin{array}{c}h_{\text {кон }}, \\
\text { мм }\end{array}$ & $\begin{array}{c}h_{0}, \\
\text { мм }\end{array}$ \\
\hline $\mathrm{Fe}_{2} \mathrm{~V}_{0.9} \mathrm{Nb}_{0.1} \mathrm{Al}$ & 3.11 & & 6.50 & 2.70 & 3.14 \\
$\mathrm{Fe}_{2} \mathrm{~V}_{0.8} \mathrm{Nb}_{0.2} \mathrm{Al}$ & 1.53 & & 6.50 & 1.33 & 2.47 \\
$\mathrm{Fe}_{2} \mathrm{VAl}_{0.9} \mathrm{Ga}_{0.1}$ & 2.05 & & 6.61 & 1.76 & 2.38 \\
$\mathrm{Fe}_{2} \mathrm{VAl}_{0.8} \mathrm{Ga}_{0.2}$ & 1.60 & 15 & 6.55 & 1.38 & 2.33 \\
$\mathrm{Fe}_{2} \mathrm{~V}_{0.9} \mathrm{Nb}_{0.1} \mathrm{Al}_{0.9} \mathrm{Ga}_{0.1}$ & 1.58 & & 6.84 & 1.31 & 2.61 \\
$\mathrm{Fe}_{2} \mathrm{~V}_{0.8} \mathrm{Nb}_{0.2} \mathrm{~A}_{l 0.8} \mathrm{Ga}_{0.2}$ & 2.19 & & 6.95 & 1.78 & 2.34
\end{tabular}

Таблица 2. Номинальный состав, масса до спекания $m_{\text {нач}}$, диаметр пресс-формы $d_{\text {пф }}$, плотность после спекания $\rho_{\text {кон }}$, высота образцов до, $h_{0}$, и после, $h_{\text {кон }}$, спекания скуттерудитов $\mathrm{Ga}_{x} \mathrm{In}_{y} \mathrm{Te}_{z} \mathrm{Co}_{4} \mathrm{Sb}_{12}(x=y=0,1, z=0,0.5,1,2,3)$

\begin{tabular}{l|c|c|c|c|c}
\hline Номинальный состав & $\begin{array}{c}m_{\text {нач, }} \\
\Gamma\end{array}$ & $\begin{array}{c}d_{\text {пф, }}, \\
\text { мм }\end{array}$ & $\begin{array}{c}\rho_{\text {кон }}, \\
\text { г/см }\end{array}$ & $\begin{array}{c}h_{\text {кон, }} \\
\text { мм }\end{array}$ & $\begin{array}{c}h_{0}, \\
\text { мм }\end{array}$ \\
\hline $\mathrm{GaInCo}_{4} \mathrm{Sb}_{12} \mathrm{Te}_{3}$ & & & 7.52 & 3.01 & 6.43 \\
$\mathrm{GaInCo}_{4} \mathrm{Sb}_{12} \mathrm{Te}_{1}$ & & & 7.52 & 3.01 & 4.55 \\
$\mathrm{CoSb}_{3}$ & & & 7.56 & 2.99 & 4.14 \\
$\mathrm{GaCo}_{4} \mathrm{Sb}_{12} \mathrm{Te}_{1}$ & 4 & 15 & 7.48 & 3.03 & 4.45 \\
$\mathrm{GaCo}_{4} \mathrm{Sb}_{12} \mathrm{Te}_{2}$ & & & 7.34 & 3.08 & 4.42 \\
$\mathrm{GaCo}_{4} \mathrm{Sb}_{12} \mathrm{Te}_{3}$ & & & 7.57 & 2.99 & 5.16 \\
$\mathrm{InCo}_{4} \mathrm{Sb}_{12} \mathrm{Te}_{3}$ & & & 7.62 & 2.97 & 4.97
\end{tabular}

Япония). Для проведения спекания порошок помещался в цилиндрическую графитовую пресс-форму с внутренним диаметром 15 мм, внешним диаметром 35 мм, высотой 50 мм. Между материалом образца, пуансонами и пресс-формой прокладывалась графитовая бумага. Для образцов на основе $\mathrm{Fe}_{2} \mathrm{VAl}$ давление при прессовании составляло $50 \mathrm{MПа,} \mathrm{а} \mathrm{для} \mathrm{скуттерудитов} \mathrm{на} \mathrm{основе}$ $\mathrm{CoSb}_{3}$ - 56 МПа. Для всех образцов давление поддерживалось постоянным в ходе всего процесса спекания вплоть до окончания выдержки образцов при температуpe спекания, после чего давление плавно опускалось до $\sim 10$ МПа. Скорость нагрева для всех образцов составляла 50 /мин (для сплавов Гейслера контролировалась по показаниям пирометра; до температуры $573^{\circ} \mathrm{C}$ нагрев осуществлялся вручную, не более $50^{\circ}$ Мин), скорость охлаждения не контролировалась. Для скуттерудитов температура спекания составляла $650^{\circ} \mathrm{C}$, а время выдержки при температуре спекания 10 мин; для сплавов Гейслера $1060^{\circ} \mathrm{C}$ и 5 мин, соответственно. Создаваемое напряжение и пропускаемый ток устанавливались автоматически, но для всех образцов не превышали 1.5 В и 1000 А соответственно.

Во время процесса спекания непрерывно фиксировались так называемые карты спекания, т.е. временны́е зависимости температуры, давления (усилия), пропус- 

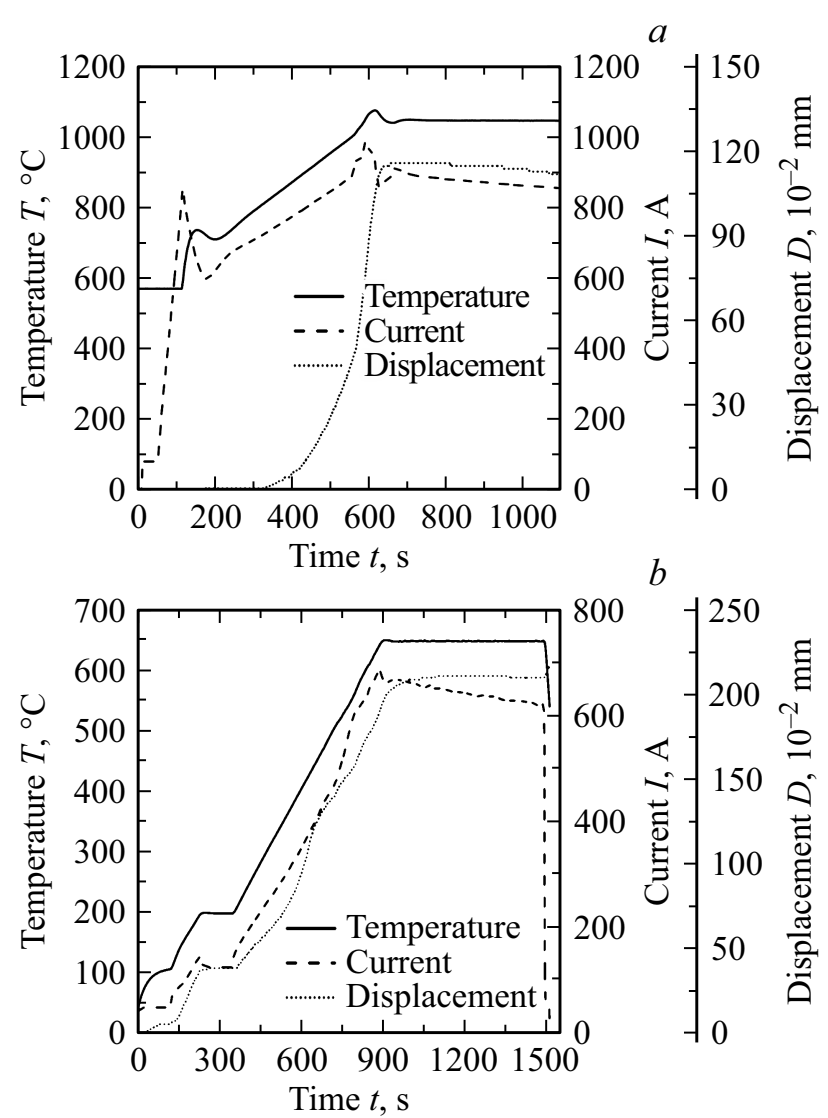

Pис. 1. Зависимость температуры, тока и усадки от времени, характерная для $(a)$ сплавов Гейслера и $(b)$ скуттерудитов

каемого через графитовую пресс-форму тока, напряжения, уровня вакуума в рабочей камере, степени и скорости уплотнения (усадки) материала образца по смещению верхнего электрода. Для измерения температуры использовалась термопара, которая вставлялась в специальное отверстие в пресс-форме; расстояние между дном отверстия и поверхностью образца составляло не более 1 мм. На рис. 1 представлены типичные временны́е зависимости температуры, значений тока, давления и усадки для сплавов Гейслера и скуттерудитов.

Вычисление параметров, характеризующих микропроцессы, протекающие в образце, было произведено в программе MS Excel. Для этого была записана функция производной пористости по времени (3) для нескольких моментов времени с шагом порядка 50-100 с в зависимости от образца. В каждый момент времени в функцию $d \theta / d t$ были подставлены мгновенные значения температуры спекания, приложенного давления, пористости. Мгновенные значения пористости рассчитывались исходя из мгновенных значений усадки и объема образца. После этого были подобраны значения параметров $Q$ и $m$, выступающие в качестве коэффициентов данной функции. После нахождения решения были получены оптимизированные значения величин $A_{m}, m$ и $Q$.

\section{4. Результаты}

Были получены графики изменения коэффициента чувствительности к скорости деформации $m$ в зависимости от времени спекания $t$ для образцов сплавов Гейслера и скуттерудитов (рис. 2).

Для образцов сплавов Гейслера в начале эксперимента значения $m$ варьировались от $\sim 0.75$ до $\sim 0.91$ и по мере процесса спекания достигали постоянного значения в пределах от 0.97 до 1, что соответствует переходу от механизма проскальзывания зерен к диффузионным процессам (рис. 2,a).

Для скуттерудитов (рис. 2,b) можно выделить три этапа уплотнения:

1. при $0.35<m<0.4$ преобладает ползучесть, контролируемая скольжением дислокаций;

2. затем при $0.4<m<0.5$ происходит постепенная смена основного механизма деформации на ползучесть при проскальзывании зерен;

3. на финальном этапе $0.5<m<0.8$ преобладает механизм диффузионной ползучести.

\section{5. Обсуждение результатов}

Сравнение полученных значений параметра $m$ для сплавов Гейслера и скуттерудитов позволяет сделать вывод о разных механизмах усадки образцов. Для скуттерудитов параметр $m$ принимает более низкие значения (от 0.35 до 0.8) и изменяется в более широком диапазоне в сравнении со значениями, полученными для сплавов Гейслера (значения $m$ от 0.75 до 1). Это может быть связано с отличием в механических свойствах материалов и большей пластичностью сплавов Гейслера. Сплавы Гейслера также обладают более высокими значениями коэффициентов тепло- и электропроводности, что может приводить к более быстрому нагреву порошка и ускорить процесс диффузии.

При этом характер зависимости параметра $m$ от времени спекания близок к зависимости температуры спекания от времени. На рис. 3 показаны нормированные кривые температуры и параметра $m$ в зависимости от времени спекания. На рисунке представлены нормированные кривые для одного образца скуттерудита и одного образца сплава Гейслера. Для всех остальных образцов других составов также прослеживалось аналогичное соответствие.

Таким образом, одним из возможных подходов в регулировании смены механизмов уплотнения может быть регулирование скорости нагрева образцов при спекании. При необходимости сохранения определенного предпочтительного механизма спекания, либо для контроля продолжительности времени действия того или иного механизма возможен подбор кривой скорости нагрева образца. Для этой цели необходимо определение механизма уплотнения, действующего на ранней стадии 
$a$

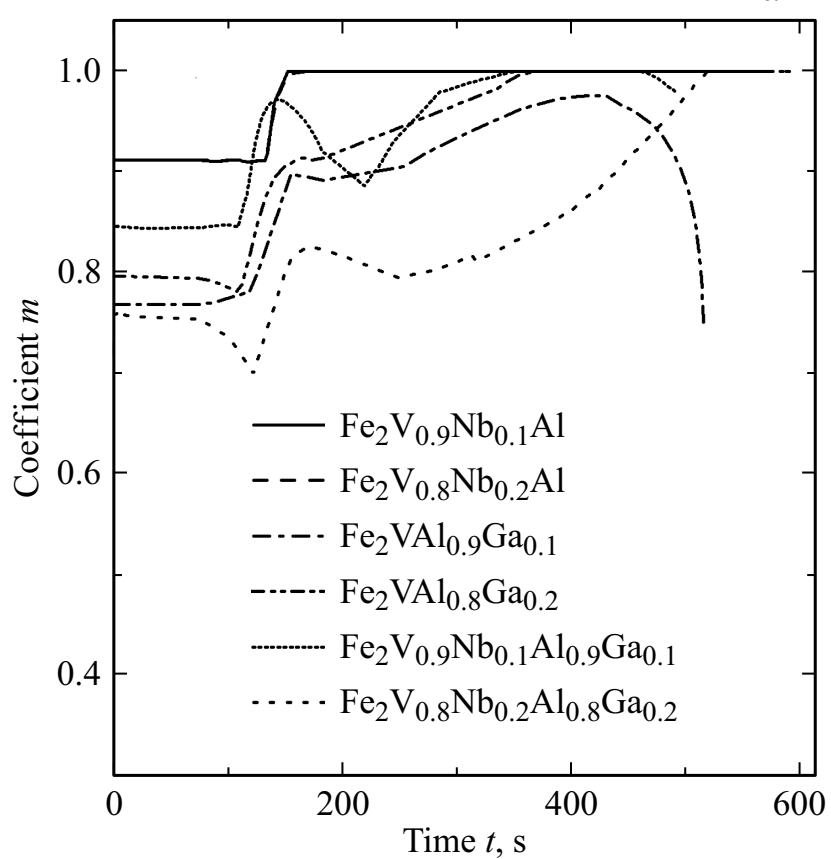

$b$

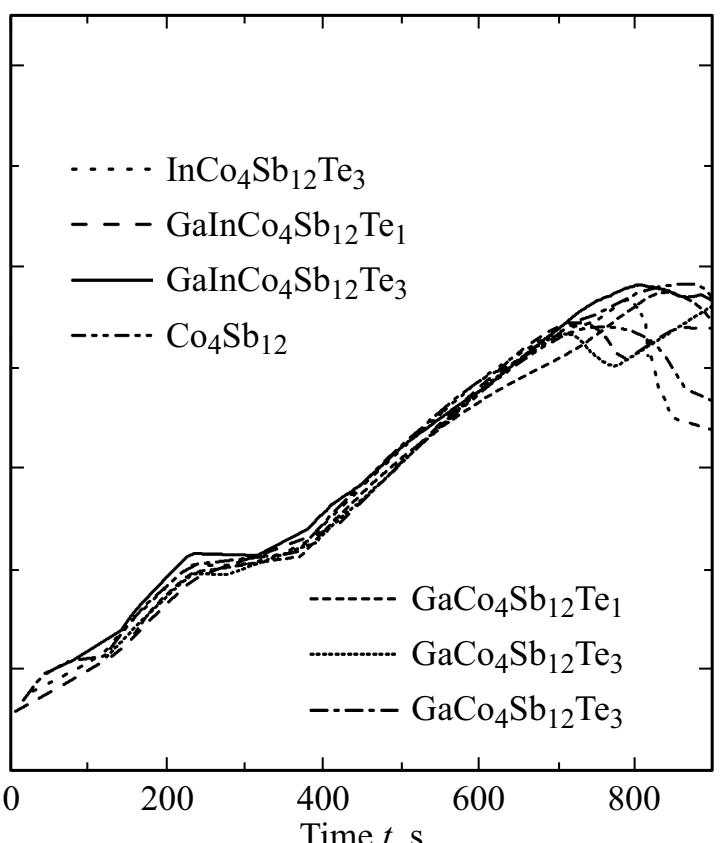

Рис. 2. График зависимости коэффициента $m$ от времени спекания для (a) сплавов Гейслера $\mathrm{Fe}_{2} \mathrm{~V}_{1-x} \mathrm{Nb}_{x} \mathrm{Al}_{1-y} \mathrm{Ga}_{y}(x=y=0,0.1$ и 0.2$)$ и $(b)$ скуттерудитов $\mathrm{Ga}_{x} \operatorname{In}_{y} \mathrm{Te}_{z} \mathrm{Co}_{4} \mathrm{Sb}_{12}(x=y=0,1, z=0,1,2,3)$
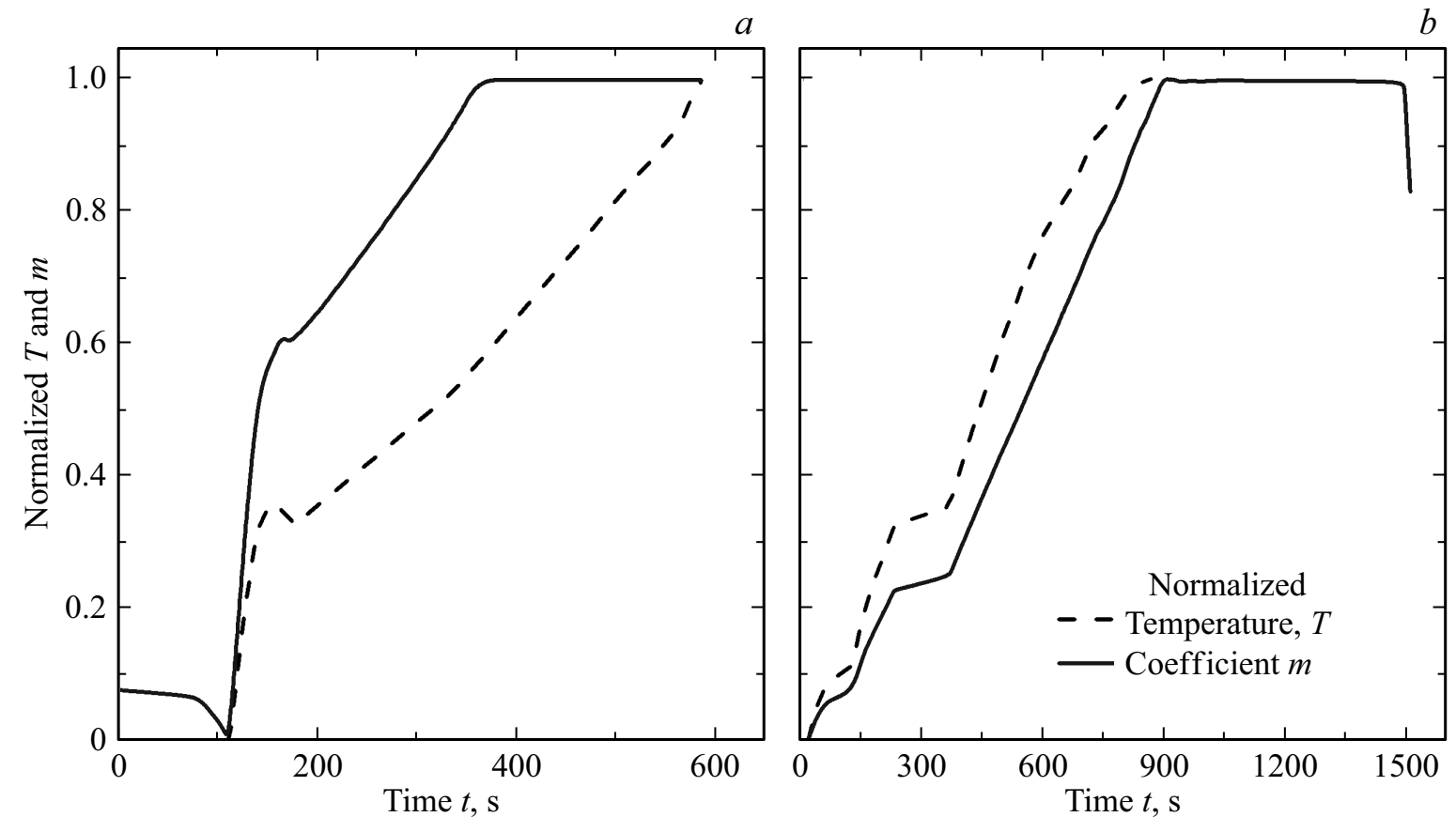

Рис. 3. Нормированные значения коэффициента $m$ и температуры для (a) $\mathrm{Fe}_{2} \mathrm{VAl}_{0.8} \mathrm{Ga}_{0.2}$ и $(b) \mathrm{GaInCo}_{4} \mathrm{Sb}_{12} \mathrm{Te}_{3}$

спекания (который не должен отличаться для образцов одного состава, обрабатываемых при одинаковом давлении). Для получения более полной картины соотношения функций $m(t)$ и $T(t)$ необходимо проведение расчетов для образцов, обрабатывающихся при разных скоростях нагрева и разных температурах спекания.
В начальный момент времени параметр $m$ для образцов сплавов Гейслера принимает разные значения. Принимая во внимание то, что температура в этот момент равна комнатной, и ток еще не проходит через образец ввиду его высокой пористости, можно сделать вывод, что начальное значение параметра $m$ зависит 
от давления и характеристик нанопорошка. В таком случае отдельно следует рассмотреть случаи для порошков различного исходного гранулометрического состава, обрабатываемых при разном давлении.

\section{6. Заключение}

Показано, что анализ кинетики спекания термоэлектрических порошков может проводиться на основе модели вязкотекучей деформации пористого тела. Для реализации подхода целесообразна аппроксимация функции производной пористости по времени по степенному закону и дальнейший анализ функции с применением метода обобщенного понижающего градиента. Используемый подход позволяет отследить изменение степенного показателя функции и соотнести его с преобладающим механизмом уплотнения порошка.

\section{Финансирование работы}

Экспериментальная часть исследования (спекание скуттерудитов) выполнена за счет гранта Российского научного фонда (проект № 19-79-10282). Моделирование выполнено при финансовой поддержке Университета ИТМО.

\section{Конфликт интересов}

Авторы заявляют, что у них нет конфликта интересов.

\section{Список литературы}

[1] O. Guillon, J. Gonzalez Julian, B. Dargatz, T. Kessel, G. Schierning, J. Räthel, M. Herrmann. Adv. Eng. Mater., 16 (7), 830 (2014).

[2] X. Wei, C. Back, O. Izhvanov, O.L. Khasanov, C.D. Haines, E.A. Olevsky. Materials, 8 (9), 6043 (2015).

[3] D.V. Dudina, B.B. Bokhonov, E.A. Olevsky. Materials, 12 (3), 541 (2019).

[4] G. Lee, M.S. Yurlova, D. Giuntini, E.G. Grigoryev, O.L. Khasanov, J. McKittrick, E.A. Olevsky. Ceram. Int., 41 (10), 14973 (2015).

[5] G. Lee, E.A. Olevsky, C. Manière, A. Maximenko, O. Izhvanov, C. Back, J. McKittrick. Acta Mater., 144, 524 (2018).

[6] M. Zhang, T. Yuan, R. Li, S. Xie, M. Wang, Q. Weng. Ceram. Int., 44 (4), 3571 (2018).

[7] C. Dong, X. Bi, J. Yu, R. Liu, Q. Zhang. J. Alloys Compd., 781, 84 (2019).

[8] Z. Trzaska, G. Bonnefont, G. Fantozzi, J.P. Monchoux. Acta Mater., 135, 1 (2017).

[9] S. Deng, R. Li, T. Yuan, S. Xie, M. Zhang, K. Zhou, P. Cao. Scripta Mater., 143, 25 (2018).

[10] C. Yang, M.D. Zhu, X. Luo, L.H. Liu, W.W. Zhang, Y. Long, E.J. Lavernia. Scripta Mater., 139, 96 (2017).

[11] F.R. Nabarro. Mater. Sci. Eng. A-struct., 387, 659 (2004).

[12] E.A. Olevsky. Mater. Sci. Eng. R. 23 (2), 41 (1998).

[13] K.R. Athul, U.T.S. Pillai, A. Srinivasan, B.C. Pai. Adv. Eng. Mater., 18 (5), 770 (2016).

\section{The analysis of thermoelectric powder compaction mechanisms within field-activated sintering of skutterudites and Heusler alloys}

A.S. Tukmakova ${ }^{1}$ N.I. Khakhilev ${ }^{1}$ D.B. Shcheglova ${ }^{1}$ V.D. Nasonov ${ }^{1}$, A.P. Novitskii ${ }^{2}$, I.A. Sergienko ${ }^{2}$, A.V. Novotelnova ${ }^{1}$

1 ITMO University, 197101 St. Petersburg, Russia

${ }^{2}$ National University of Science and Technology „MISIS“, 119049 Moscow, Russia

Abstract The analysis of the shrinkage rate of powders, based on the power law creep model of a porous body, was carried out in this paper to calculate the compaction parameters of $\mathrm{CoSb}_{3}$-based skutterudites and $\mathrm{Fe}_{2} \mathrm{VAl}$-based Heusler alloys within field-activated sintering. It was indicated that this method, which had already been used for metal and ceramic powders, is applicable for thermoelectric powders. The values of strain rate sensitivity were obtained, and the corresponding powder compaction mechanisms have been defined. The main creep mechanism for skutterudites was found to be a dislocation climb, that later was replaced by grain boundary sliding, and the last sintering stage was associated with diffusional creep. The main creep mechanism for Heusler alloys was grain boundary sliding, later replaced by diffusional creep. 